Marjan Asemani and Navideh Anarjan*

\title{
Green synthesis of copper oxide nanoparticles using Juglans regia leaf extract and assessment of their physico-chemical and biological properties
}

https://doi.org/10.1515/gps-2019-0025

Received November 8, 2018; accepted March 27, 2019.

\begin{abstract}
Copper oxide nanoparticles (CuO NPs) were green synthesized using walnut leaf extract. Effects of three synthesis parameters namely; amount copper salt (1-4 g), amount of walnut leaf extract $(10-40 \mathrm{~mL})$ and furnace temperature $\left(300-500^{\circ} \mathrm{C}\right)$, on the particle size as manifested in broad absorption peak $\left(\lambda_{\max }, \mathrm{nm}\right)$, concentration (absorbance), antioxidant activity and antibacterial activity as minimum inhibitory concentration (MIC) of the fabricated $\mathrm{CuO}$ NPs were studied using response surface methodology, based on Box behnken experimental design. The spherical and crystalline monodispersed fabricated $\mathrm{CuO}$ NPs with mean particle size of $80 \mathrm{~nm}$, were achieved using optimum synthesis parameters including $1 \mathrm{~g}$ copper salt, $14 \mathrm{~mL}$ walnut leaf extract and $490^{\circ} \mathrm{C}$ of furnace temperature. The fabricated $\mathrm{CuO}$ NPs at these conditions had maximum antioxidant activity of $83.64 \%$ and minimum MIC value of $1.78 \% \mathrm{w} / \mathrm{v}$ against $E$. coli, with $\lambda_{\max }$ and absorbance values of $226 \mathrm{~nm}, 4.44 \%$ a.u., respectively.
\end{abstract}

Keywords: copper oxide nanoparticles; walnut leaf extract; green synthesis; antioxidant activity; minimum inhibitory concentration

\section{Introduction}

Among metal oxide, copper oxide has gained more attention in the last decade, due to its distinctive properties such as high temperature superconductivity, spin dynamics and electron correlation effects, which

\footnotetext{
* Corresponding author: Navideh Anarjan, Faculty of Engineering, Tabriz Branch, Islamic Azad University, East Azarbaijan, Tabriz, Iran; Department of basic science, Biotechnology Research Center, Tabriz Branch, Islamic Azad University, East Azarbaijan, Tabriz, Iran, e-mail: anarjan@iaut.ac.ir and anarjan@gmail.com

Marjan Asemani, Faculty of Chemical Engineering, Tabriz Branch, Islamic Azad University, East Azarbaijan, Tabriz, Iran
}

those increase widely its applications as heterogeneous catalysts, antioxidants, drug delivery agents, and imaging agents in field of biomedicine [1,2]. Furthermore, copper oxide is inexpensive antimicrobial agent when compared to inorganic bactericidal agents such as silver and gold, and has longer shelf life as compared to other organic antimicrobial agents [1]. All the mentioned properties of copper oxide drastically increase when it fabricates in nano-size scale. In fact, copper oxide nanoparticles (CuO NPs) due to their extremely unusual crystal morphologies and high surface area to volume ratio, have unique physical, chemical and biological properties $[1,3]$.

Development of a non-toxic, clean, reliable, costeffective, eco-friendly and biocompatible processes to NPs fabrication is one of the great interests of researchers in the last decade to replace them with common physical and chemical synthesis methods, which those are high energy consuming with intensive capital cost and utilize toxic chemicals and non-polar solvents in their processes [4]. Green synthesis of NPs with plants and their derivatives extract, have gained more attentions during last years, due to the presence of the numerous natural reductants (i.e., polyphenols, flavonoids, tannins, ascorbic acids and sugars) and stabilizers (i.e., proteins, carbohydrates, gums and pectic substances) [5-7]. Numerous researches have been done on green synthesis of $\mathrm{CuO}$ NPs with plant extract such as tea leaf, coffee powder and Eichhornia crassipes extracts $[8,9]$.

Walnut (- regia L.) leaf contains numerous main bioactive compounds such as, malic acid, 3- $O$-caffeoylquinic acids, quercetin $O$-pentoside, sucrose, disaccharide, $\alpha$-tocopherol and tocopherol isomer, and phenolic compounds which those can be effectively utilized as reducing and stabilizing mediators in the NPs production such as silver NPs [10].

Present study focused on i) possible application of the walnut leaf extract in the CuO NPs fabrication, ii) optimization of the synthesis process using response surface methodology to achieved CuO NPs with appropriate physico-chemical attributes, and iii) valuation of antibacterial activities of the formed $\mathrm{CuO}$ NPs using walnut leaf extract. 


\section{Materials and method}

Walnut fresh leaves, same in size and colour, and free of microbial damages, were collected locally from a walnut garden in Tabriz, Iran. Copper nitrate, $\mathrm{Cu}\left(\mathrm{NO}_{3}\right)_{2}$, Dimethyl sulfoxide (DMSO), methanol, ethanol, nutrient agar and Mueller Hinton broth were provided from Merck Company (Merck, Darmstadt, Germany). Resazurin reagent was purchased from (Sigma-Aldrich, United State of America). E. coli (PPTCC 1270) was obtained from the microbial Persian type culture collection (PTCC, Tehran, Iran). All the solutions in the present study were prepared using deionized double distilled water (DI).

\subsection{Preparation of walnut leaf extract and synthesis of CuO NPs}

Provided walnut leaves were washed three time using DI water to remove their surface contaminations and shade dried at room temperature $\left(30^{\circ} \mathrm{C}\right)$ during a week. A domestic miller (MX-GX1521; Panasonic, Tokyo, Japan) was utilized to prepare walnut dried powder and in order to prepare walnut leaf extract, $5 \mathrm{~g}$ dried powder was added into $100 \mathrm{~mL}$ of boiling DI water for $1 \mathrm{~h}$, and after cooling the mixture solution, it was filtered (Whatman No. 40 filter paper) and the extract was then collected in dark bottle and kept in the refrigerator.

In order to prepare $\mathrm{CuO} \mathrm{NPs}, 1$ to $4 \mathrm{~g}$ of the copper nitrate was added into the defined amount of the prepared walnut leaf extract (10-40 mL) and the reaction solutions were mixed using a heater-stirred, adjusted at $500 \mathrm{rpm}$ and $70^{\circ} \mathrm{C}$, for $15 \mathrm{~min}$ and finally the samples were put in an electric furnace (FM4P, Fanazma Gostar Co., Alborz, Iran) adjusted at 300 to $500^{\circ} \mathrm{C}$ for $2 \mathrm{~h}$. The obtained powders, as farmed CuO NPs, were then used for further studies.

\subsection{Physico-chemical properties of the synthesized CuO NPs}

Formation of the $\mathrm{CuO}$ NPs can be easily confirmed using UV-Vis spectrophotometry (UV-3600 Plus, Shimadzu Co., Tokyo, Japan) because of the surface Plasmon resonance (SPR) of the formed NPs. Due to the SPR of the fabricated $\mathrm{CuO}$ NPs, the solution containing CuO NPs indicated the broad absorption peak $\left(\lambda_{\max }\right)$ in the wavelength ranged 200-300 nm [8]. Furthermore, fabrication of CuO NPs can be easily verified by X-Ray Diffractometry (XRD: D5000, Siemens Co. Germany) using $\mathrm{Cu}$ Ka radiation and compression of the obtained pattern with the standard
XRD pattern for $\mathrm{CuO}$ NPs. Scanning electron microscopy (SEM, CamScan MV 2300, Tescan, Czech Republic) was utilized to assess of the structural properties and morphology of the formed CuO NPs. Antioxidant activity of the fabricated $\mathrm{CuO}$ NPs using walnut leaf extract had been assessed according to the scavenging ability on 2,2-diphenyl-2-picrylhydrazyl (DPPH) method, which that was described by Vahidi et al. [11].

\subsection{Antibacterial activity assess of the formed CuO NPs}

Antibacterial activity of the formed $\mathrm{CuO}$ NPs was assessed using MIC test according to the clinical and laboratory standards institute (CLSI) protocol [12]. In order to obtain the MIC values, serial dilutions of $\mathrm{CuO}$ NPs with the concentrations of 100, 50, 25, 6.25, 3.12, 1.56, 0.78 and $0.39 \%(\mathrm{~W} / \mathrm{V})$ were provided in a 96 well-cell plate. After that 100 and $30 \mu \mathrm{L}$ of the sterilized MHB and Resazurin reagent were added into the wells, respectively. Finally, $100 \mu \mathrm{L}$ provided bacteria suspension (E. coli) adjusted to 0.5 McFarland standards have been added into the wells. The turbidity of the poured wells were measured at wavelength of $625 \mathrm{~nm}$ by UV-visible spectroscopy measurements (250-800 nm, Perkn Elmer, Germany), at the beginning of the test and after $24 \mathrm{~h}$ incubation of the 96 well-cell plate at $37^{\circ} \mathrm{C}$ and the MICs were determined.

\subsection{Design of experiments and data analysis}

Response surface methodology (RSM) has numerous advantages as compared to other statistical techniques due to its potential to generate valuable data using minimum experiment runs and assessment of their different effects such as linear, quadratic and interaction on the responses [13-16]. Therefore, Box behnken design, using a block, with RSM were utilized to experimental design and optimize of the CuO NPs synthesis parameters namely; amount of copper ion concentration $\left(\mathrm{X}_{1}, 1-4 \mathrm{~g}\right)$, amount of walnut leaf extract $\left(\mathrm{X}_{2}, 10-40 \mathrm{~mL}\right)$ and electric furnace temperature $\left(\mathrm{X}_{3}, 300-500^{\circ} \mathrm{C}\right)$, on the $\lambda_{\max }\left(\mathrm{Y}_{1}, \mathrm{~nm}\right)$, absorbance at $\lambda_{\max }\left(\mathrm{Y}_{2}, \%\right.$ a.u.), MIC $\left(\mathrm{Y}_{3}\right)$, and anti-oxidant activity $\left(\mathrm{Y}_{4}, \%\right)$ of the mixture solutions containing $\mathrm{CuO}$ NPs. Response variables, as function of the synthesized parameters, were followed a second-order polynomial Eq. 1, which in this equation, $B_{0}$ is a constant and $B_{i}, B_{i i}$ and $B_{i j}$ are the coefficients of the linear, quadratic and interaction terms. 
Table 1: Box behnken design and response variables for synthesis of CuO NPs using walnut leaf extract.

\begin{tabular}{|c|c|c|c|c|c|c|c|}
\hline Sample runs & $\begin{array}{r}\text { Ion concentration } \\
\text { (g) }\end{array}$ & $\begin{array}{l}\text { Leaf extract } \\
\text { amount }(\mathrm{mL})\end{array}$ & $\begin{array}{r}\text { Temperature } \\
\left({ }^{\circ} \mathrm{C}\right)\end{array}$ & $\begin{array}{r}\lambda_{\max } \\
(\mathrm{nm})\end{array}$ & $\begin{array}{r}\text { Absorbance } \\
\text { (\% a.u.) }\end{array}$ & $\begin{array}{r}\text { Antioxidant } \\
(\%)\end{array}$ & $\begin{array}{r}\text { MIC } \\
(\% \mathrm{w} / \mathrm{v})\end{array}$ \\
\hline 1 & 2.5 & 10 & 500 & 237.5 & 3.000 & 86.0 & 4.100 \\
\hline 2 & 1.0 & 10 & 400 & 245.0 & 2.410 & 88.7 & 2.310 \\
\hline 3 & 4.0 & 25 & 500 & 260.0 & 1.100 & 88.1 & 1.470 \\
\hline 4 & 2.5 & 10 & 300 & 246.0 & 1.314 & 86.3 & 2.040 \\
\hline 5 & 4.0 & 25 & 300 & 250.0 & 0.500 & 85.7 & 3.000 \\
\hline 6 & 2.5 & 25 & 400 & 241.0 & 1.066 & 85.9 & 1.140 \\
\hline 7 & 2.5 & 40 & 500 & 260.0 & 2.300 & 83.4 & 1.100 \\
\hline 8 & 2.5 & 40 & 300 & 258.0 & 1.188 & 78.8 & 1.520 \\
\hline 9 & 4.0 & 40 & 400 & 260.0 & 2.009 & 90.0 & 1.670 \\
\hline 10 & 1.0 & 25 & 500 & 225.0 & 5.010 & 80.0 & 1.095 \\
\hline 11 & 1.0 & 40 & 400 & 260.0 & 4.338 & 79.7 & 1.390 \\
\hline 12 & 2.5 & 25 & 400 & 290.0 & 1.070 & 88.0 & 1.410 \\
\hline 13 & 1.0 & 25 & 300 & 267.0 & 1.100 & 83.7 & 1.250 \\
\hline 14 & 2.5 & 25 & 400 & 263.0 & 1.000 & 87.3 & 2.030 \\
\hline 15 & 4.0 & 10 & 400 & * & * & * & \\
\hline
\end{tabular}

* Out of range.

$$
\begin{aligned}
\mathrm{Y}= & \beta_{0}+\beta_{1} \mathrm{X}_{1}+\beta_{2} \mathrm{X}_{2}+\beta_{3} \mathrm{X}_{3}+\beta_{11} \mathrm{X}_{1}^{2}+\beta_{22} \mathrm{X}_{2}^{2}+ \\
& \beta_{33} \mathrm{X}_{3}^{2}+\beta_{12} \mathrm{X}_{1} \mathrm{X}_{2}+\beta_{13} \mathrm{X}_{1} \mathrm{X}_{3}+\beta_{23} \mathrm{X}_{2} \mathrm{X}_{3}
\end{aligned}
$$

Analysis of variance (ANOVA) based on coefficient of determination $\left(\mathrm{R}^{2}\right)$ and the terms $\mathrm{p}$-value $(\mathrm{p}<0.05)$ and $\mathrm{F}$ ratio was employed to significance determinations of the obtained reduced models [17]. In the reduced models, those terms which had insignificant effects on the responses were removed $[18,19]$. Two-dimensional contour plots, based on the generated models, were established only for significant $(p<0.05)$ interaction effects of the synthesis parameters. Numerical multiple response optimization was utilized to obtain exact values of the optimum synthesis parameters [20]. Obtained optimum synthesis parameters were used three times to synthesis $\mathrm{CuO}$ NPs for verification of the generated reduced models accuracy [21]. Minitab v.16 statistical software (Minitab Inc., PA, USA) was used for the design of experiments, RSM, ANOVA and optimization procedures.

\section{Results and discussion}

\subsection{Generation of response surface models}

Based on the attained values for the responses (Table 1) the reduced models for the $\lambda_{\max }$, absorbance, antioxidant activity and MIC of the prepared CuO NPs, as function of copper salt, walnut leaf extract and furnace temperature were generated. Table 2 indicates regression coefficients
Table 2: Regression coefficients, $R^{2}$, adjusted $R^{2}\left(R^{2}\right.$-adj) and probability values for the final reduced models.

\begin{tabular}{lrrrr}
\hline Regression coefficient & $\begin{array}{r}\lambda_{\text {max }} \\
(\mathrm{nm})\end{array}$ & $\begin{array}{r}\text { Absorbance } \\
(\% \text { a.u. })\end{array}$ & $\begin{array}{r}\text { Antioxidant } \\
(\%)\end{array}$ & $\begin{array}{r}\text { MIC } \\
(\% \text { w/v) }\end{array}$ \\
\hline$\beta_{0}$ (constant) & 283.29 & 0.512 & 87.060 & 1.445 \\
$\beta_{1}$ (main effect) & 9.13 & -0.448 & 2.690 & 0.683 \\
$\beta_{2}$ (main effect) & $\mathrm{NS}$ & $\mathrm{NS}$ & -2.365 & -0.931 \\
$\beta_{3}$ (main effect) & -14.19 & 0.475 & 0.375 & $\mathrm{NS}$ \\
$\beta_{11}$ (quadratic effect) & $\mathrm{NS}$ & $\mathrm{NS}$ & $\mathrm{NS}$ & 0.636 \\
$\beta_{22}$ (quadratic effect) & $\mathrm{NS}$ & $\mathrm{NS}$ & $\mathrm{NS}$ & 0.573 \\
$\beta_{33}$ (quadratic effect) & -19.72 & 1.838 & -3.060 & $\mathrm{NS}$ \\
$\beta_{12}$ (interaction effect) & $\mathrm{NS}$ & $\mathrm{NS}$ & 2.295 & -0.793 \\
$\beta_{13}$ (interaction effect) & 13.00 & -1.350 & 1.525 & $\mathrm{NS}$ \\
$\beta_{21}$ (interaction effect) & $\mathrm{NS}$ & $\mathrm{NS}$ & $\mathrm{NS}$ & $\mathrm{NS}$ \\
$\beta_{23}$ (interaction effect) & $\mathrm{NS}$ & $\mathrm{NS}$ & 1.225 & $\mathrm{NS}$ \\
$\mathrm{R}^{2}$ & 0.8828 & 0.8440 & 0.9522 & 0.8665 \\
$\mathrm{R}^{2}$-adj & 0.8359 & 0.7815 & 0.8964 & 0.7831 \\
Lack of fit & 0.331 & 0.602 & 0.503 & 0.604 \\
\hline
\end{tabular}

NS: Non-significant.

1: Ion concentration (g); 2: Leaf extract amount $(\mathrm{mL})$;

3: Temperature $\left({ }^{\circ} \mathrm{C}\right)$.

of the models terms including main, quadratic and interaction, $\mathrm{R}^{2}$ and $\mathrm{R}^{2}$-adj, and lack of fit for the fitted models. As can be observed in Table 2, higher values of the $R^{2}(>0.88), R^{2}$-adj ( $>0.78$ ) and lack of fit $p$-value $(p>0.05)$ for the generated models, verified their proper precision $[22,23]$. The significance probability of the regression coefficients for the final reduced models presents in Table 3. As can be shown in Table 3, main effect of the copper salt had significant $(p<0.05)$ effects on the all studied responses. Furthermore, main and quadratic effects of furnace temperature and its 
Table 3: The significance probability ( $p$-value) of regression coefficients for the final reduced models.

\begin{tabular}{lrrrr}
\hline Terms & $\begin{array}{r}\lambda_{\max } \\
(\mathbf{n m})\end{array}$ & $\begin{array}{r}\text { Absorbance } \\
(\% \text { a.u. })\end{array}$ & $\begin{array}{r}\text { Antioxidant } \\
(\%)\end{array}$ & $\begin{array}{r}\text { MIC } \\
(\% \mathrm{w} / \mathrm{v})\end{array}$ \\
\hline$\beta_{0}$ (constant) & 0.000 & 0.046 & 0.000 & 0.000 \\
$\beta_{1}$ (main effect) & 0.007 & 0.032 & 0.001 & 0.002 \\
$\beta_{2}$ (main effect) & $\mathrm{NS}$ & $\mathrm{NS}$ & 0.002 & 0.000 \\
$\beta_{3}$ (main effect) & 0.000 & 0.028 & 0.042 & $\mathrm{NS}$ \\
$\beta_{11}$ (quadratic effect) & $\mathrm{NS}$ & $\mathrm{NS}$ & $\mathrm{NS}$ & 0.013 \\
$\beta_{22}$ (quadratic effect) & $\mathrm{NS}$ & $\mathrm{NS}$ & $\mathrm{NS}$ & 0.020 \\
$\beta_{33}$ (quadratic effect) & 0.001 & 0.000 & 0.003 & $\mathrm{NS}$ \\
$\beta_{12}$ (interaction effect) & $\mathrm{NS}$ & $\mathrm{NS}$ & 0.021 & 0.009 \\
$\beta_{13}$ (interaction effect) & 0.007 & 0.002 & 0.036 & $\mathrm{NS}$ \\
$\beta_{23}$ (interaction effect) & $\mathrm{NS}$ & $\mathrm{NS}$ & 0.045 & $\mathrm{NS}$ \\
\hline
\end{tabular}

NS: Non-significant.

1: Ion concentration $(\mathrm{g}) ; 2$ : Leaf extract amount $(\mathrm{mL})$;

3: Temperature $\left({ }^{\circ} \mathrm{C}\right)$.

interaction with copper salt had significant $(\mathrm{p}<0.05)$ effects on the $\lambda_{\max }$, absorbance and antioxidant activity of the prepared CuO NPs.

\subsection{Effects of the synthesized parameters on the $\lambda_{\max }$ and absorbance of the samples}

As can be realized in Table $1, \lambda_{\max }$ and absorbance of the prepared solutions containing $\mathrm{CuO}$ NPs varied from 225-290 $\mathrm{nm}$ and 0.500-5.010\% a.u., respectively. Obtained results revealed that $\mathrm{CuO}$ NPs with smallest particle size $\left(\lambda_{\max }\right.$ of $\left.225 \mathrm{~nm}\right)$ and maximum concentration $(5.010 \%$ a.u.) were fabricated using synthesized parameters including amount of copper salt, amount of walnut leaf extract and furnace temperature of $1 \mathrm{~g}, 25 \mathrm{~mL}$ and $500^{\circ} \mathrm{C}$, respectively. Furthermore, statistical analysis demonstrated that the interaction effects of the copper salt and furnace temperature had significant $(p<0.05)$ effects on the $\lambda_{\max }$ and absorbance of the prepared solutions containing $\mathrm{CuO}$ NPs (Table 3). Effects of the copper salt and furnace temperature on the $\lambda_{\max }$ and absorbance are indicated in Figures $1 \mathrm{a}$ and $1 \mathrm{~b}$, respectively. In the synthesis of organic NPs, the main aim is to form NPs with minimum particle size and maximum concentration which these two parameters can be manifested in minimum $\lambda_{\max }$ and maximum absorbance [7]. As can be seen in Figures $1 \mathrm{a}$ and $1 \mathrm{~b}$, $\mathrm{CuO}$ NPs with minimum $\lambda_{\max }$ and maximum absorbance could be achieved using minimum amount of copper salt and maximum furnace temperature. The obtained results can be explained by the fact that at minimum ion concentration, due to higher concentration of bioreductant compounds of the plant extract, the nucleation rate was increased and NPs were fabricated rapidly and stabilized by the presented stabilizers of the plant leaf extract $[4,5]$. This result was in line with finding of Eshghi et al., they fabricated silver NPs using walnut leaf extract [10].

\subsection{Effects of the synthesized parameters on the antioxidant activity of the samples}

As can be seen in Table 1, antioxidant activity of the fabricated $\mathrm{CuO}$ NPs is varied from 78.8 to $90 \%$. The achieved results demonstrated that maximum antioxidant activity of the fabricated $\mathrm{CuO}$ NPs was obtained using synthesis conditions including amount of copper salt, amount of walnut leaf extract and furnace temperature of $4 \mathrm{~g}, 40 \mathrm{~mL}$ and $400^{\circ} \mathrm{C}$, respectively. In addition to, statistical analysis revealed that the interaction effects of all three synthesis parameters had significant $(p<0.05)$ effects on the antioxidant activity of the formed CuO NPs using walnut leaf extract (Table 3). Effects of the synthesis parameters on the antioxidant activity of the formed CuO NPs indicate in Figures 2a-c. As clearly observed in Figure $2 \mathrm{a}$ and $2 \mathrm{~b}$, at minimum amount of copper salt, by increasing the amount of walnut leaf extract and furnace temperature, the fabricated $\mathrm{CuO}$ NPs indicated maximum antioxidant activity, respectively. The obtained results can be explained by the fact that, at lower amount of copper salt, by addition of higher amount of walnut leaf extract and subjected the prepared reaction solution into the higher temperatures, the $\mathrm{CuO}$ NPs with minimum particle size and maximum concentration were fabricated, as can be seen in Figure 1, which those increased antioxidant activity of the formed NPs [11]. However, as can be seen in Figure 2c, the formed $\mathrm{CuO}$ NPs with highest antioxidant activity was produced using maximum amount of walnut leaf extract and minimum furnace temperature.

\subsection{Effects of the synthesized parameters on the MIC of the formed CuO NPs}

As can be seen in Table 1, MIC of the formed CuO NPs is changed from 1.095 to $4.100 \% \mathrm{w} / \mathrm{v}$. The attained results demonstrated that minimum MIC of the formed $\mathrm{CuO}$ NPs was gained using synthesis conditions including amount of copper salt, amount of walnut leaf extract 



\section{A}
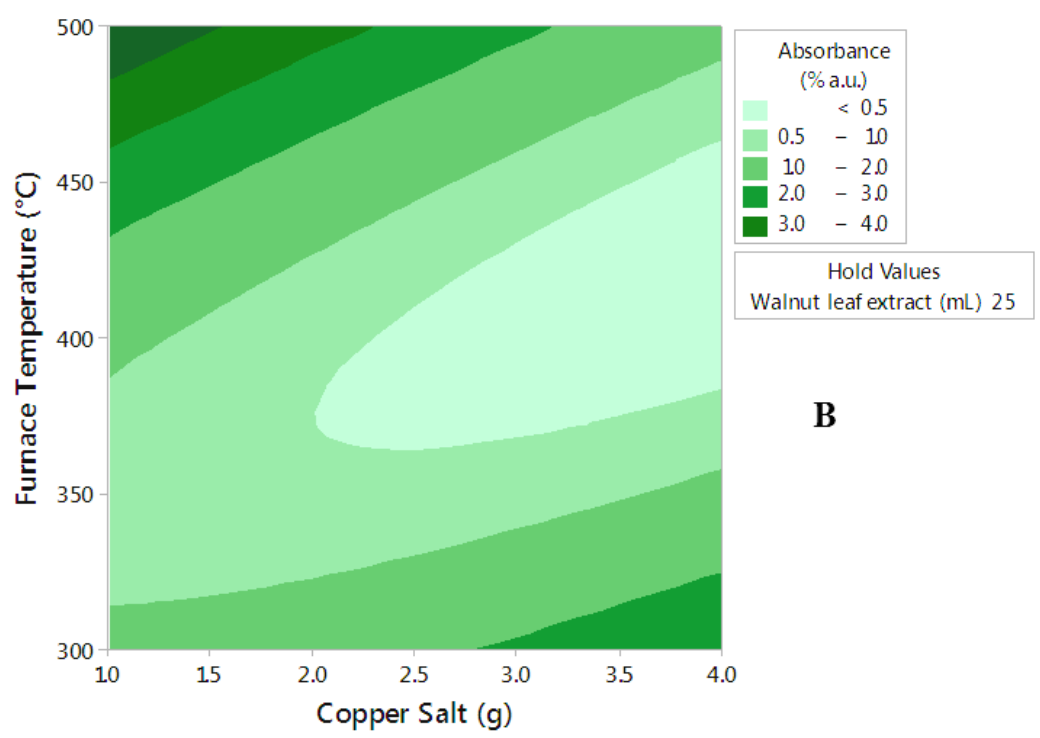

B

Figure 1: Contour plots for $\lambda \max (\mathrm{a})$ and absorbance (b) of the formed CuO NPs as function of copper salt amount (g), amount of walnut leaf extract $(\mathrm{mL})$ and furnace temperature $\left({ }^{\circ} \mathrm{C}\right)$.

and furnace temperature of $1 \mathrm{~g}, 25 \mathrm{~mL}$ and $500^{\circ} \mathrm{C}$, respectively. In addition to, statistical analysis revealed that the interaction of amounts of copper salt and walnut leaf extract had significant $(p<0.05)$ effect on the MIC of the fabricated CuO NPs using walnut leaf extract (Table 3). Furthermore, as clearly indicated in Figure 3, minimum MIC was attained by minimum and low amounts of copper salt and walnut leaf extract, respectively. This result could be related to the smallest particle size of the CuO NPs using walnut extract at minimum amount of the copper salt. Due to higher surface to volume ratio of the fabricated small NPs, those effectively attached to the microbial membrane and altered its permeability which in turn, caused microorganism death [14].

\subsection{Optimization of processing parameters for the CuO NPs synthesis}

The CuO NPs synthesized parameters were considered as optimum conditions when the formed NPs had minimum particle size (according to the $\lambda_{\max }$ ) and MIC, and maximum concentration (absorbance) and antioxidant activity. Figures 4a-c indicate graphical optimization area, white coloured surfaces, based on an overlaid contour 


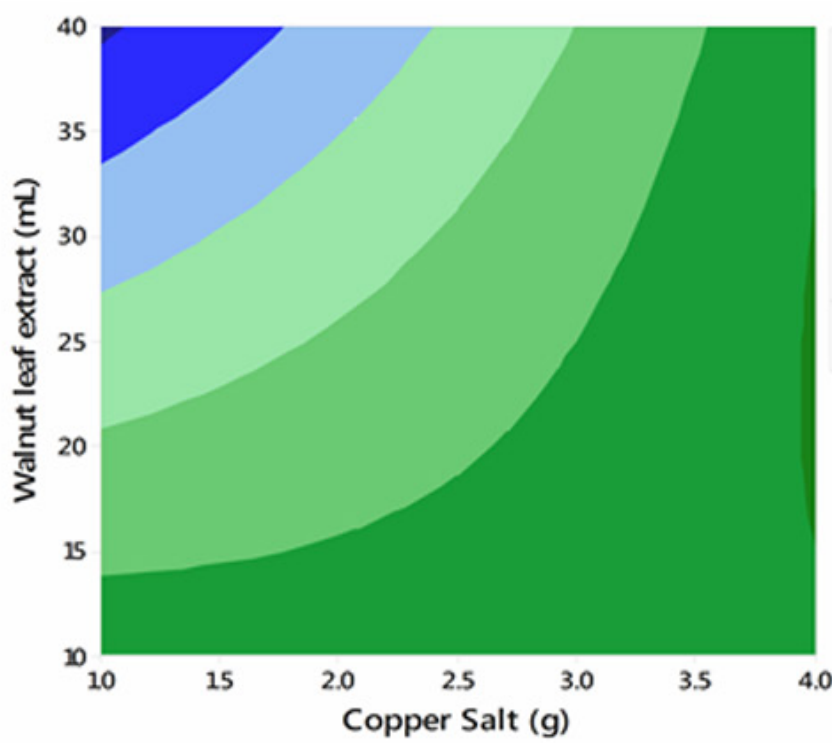

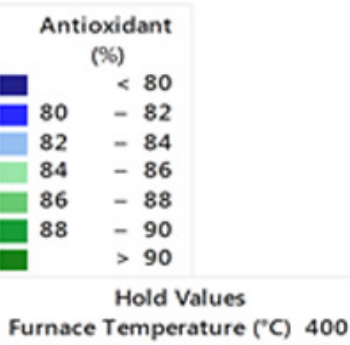

A


Walnut leaf extract $(\mathrm{mL}) 25$

B

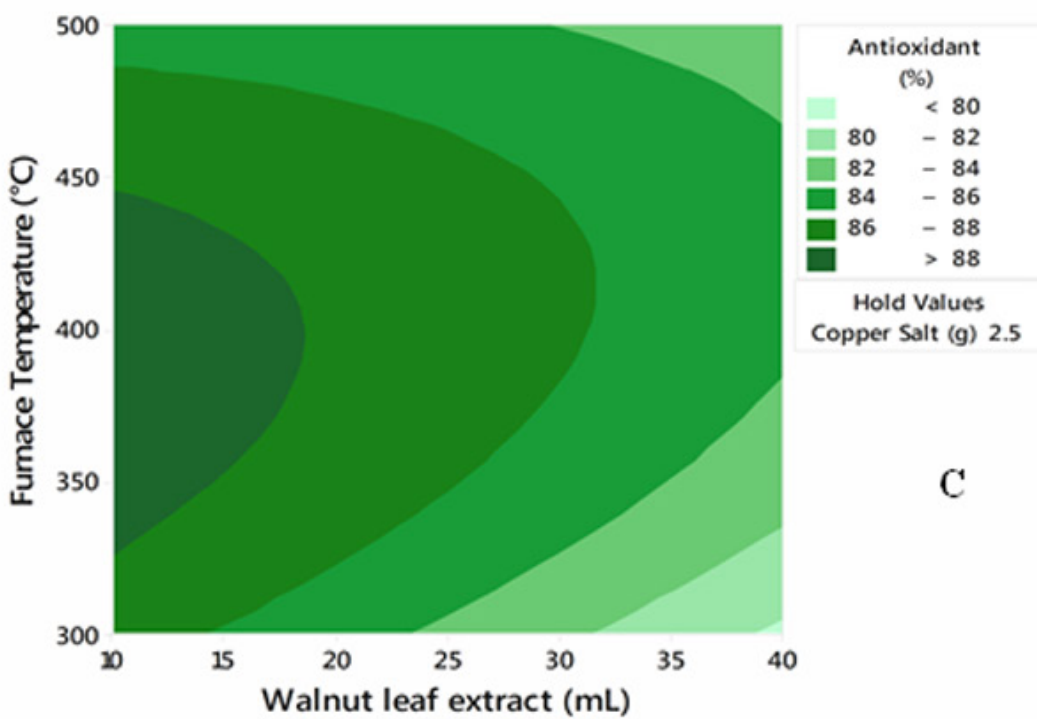

Figure 2: Contour plots for antioxidant activity of the formed CuO NPs as function of copper salt and walnut leaf extract amounts (a), copper salt amount and furnace temperature (b), and amount of copper salt and furnace temperature (c). 
plot. Numerical multiple optimization also shown that the optimum synthesized conditions for achievement of $\mathrm{Cu} \mathrm{O}$ NPs with desirable properties were obtained using $1 \mathrm{~g}$ of copper salt, $14 \mathrm{~mL}$ walnut leaf extract and $490^{\circ} \mathrm{C}$ of furnace temperature. At these obtained synthesized parameters, the values of $\lambda_{\max }$, absorbance, antioxidant activity and MIC of the fabricated CuO NPs were predicted as $226 \mathrm{~nm}$, $4.44 \%$ a.u., $83.64 \%$ and $1.78 \% \mathrm{w} / \mathrm{v}$, respectively. Statistical analysis indicated that there were non-significant differences between the experimental and predicted



Figure 3: Contour plots for MIC of the formed CuO NPs as function of copper salt amount (g), amount of walnut leaf extract (mL) and furnace temperature $\left({ }^{\circ} \mathrm{C}\right)$.

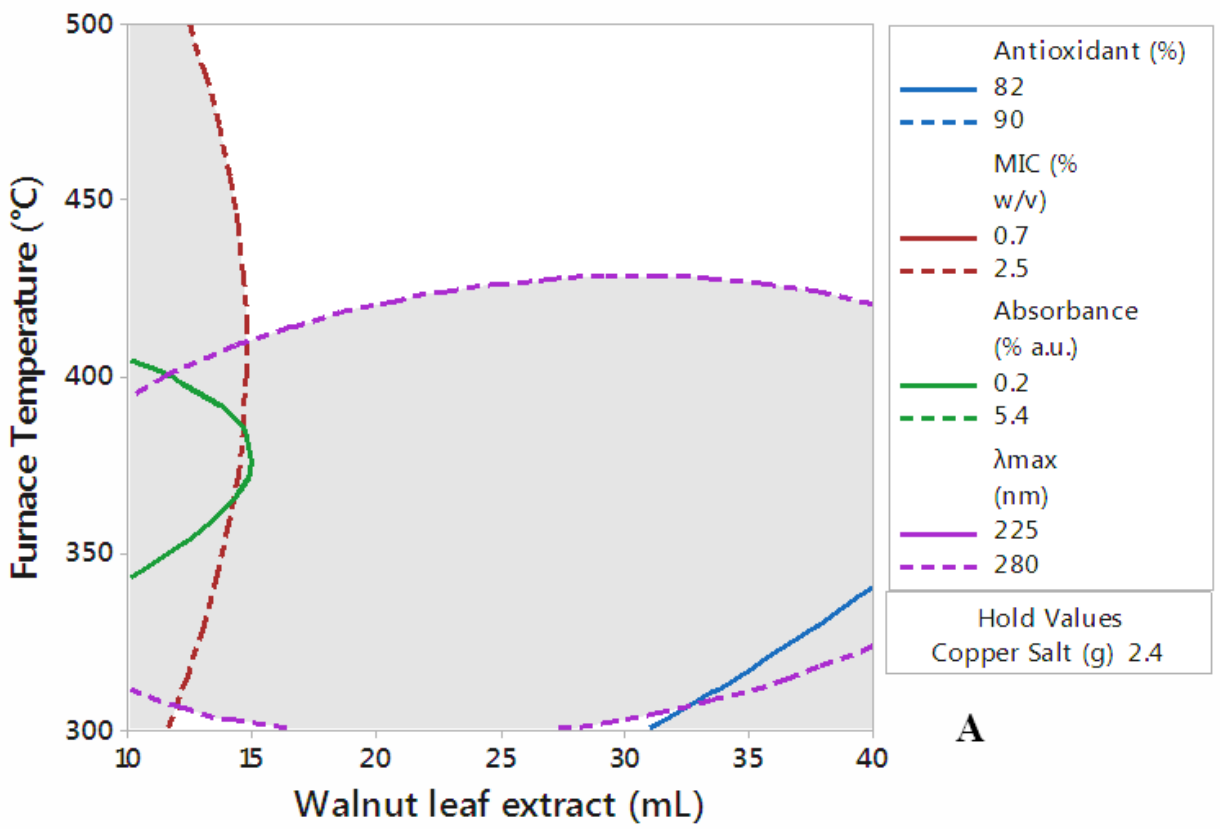

Figure 4: (Continued). 

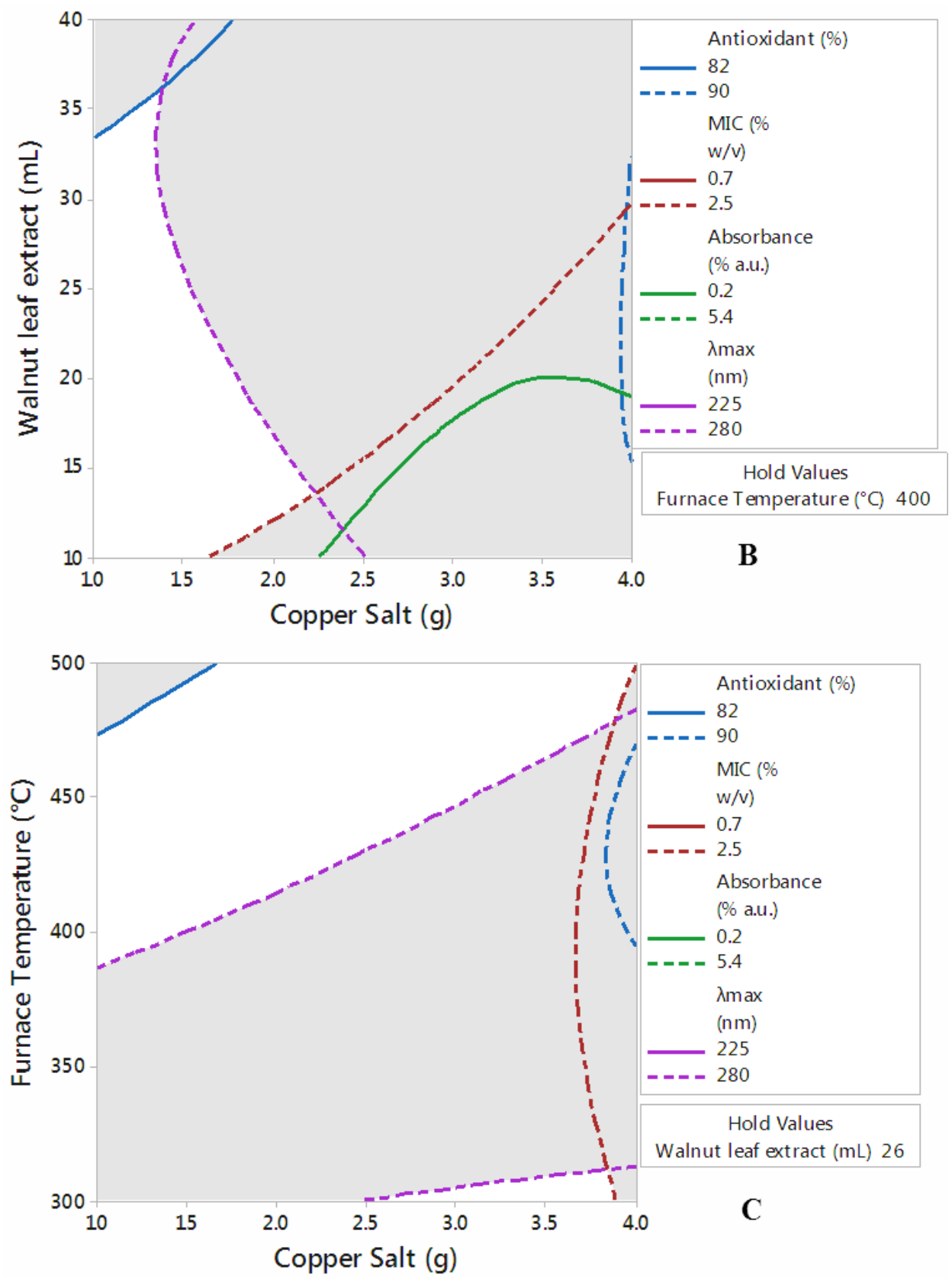

Figure 4: (a-c) Overlaid contour plot CuO NPs $\lambda$ max, absorbance, antioxidant activity and MIC with acceptable levels as function of copper salt amount $(\mathrm{g})$, amount of walnut leaf extract $(\mathrm{mL})$ and furnace temperature $\left({ }^{\circ} \mathrm{C}\right)$.

values of the responses at obtained optimum synthesized conditions. This result confirmed the adequacy of the generated reduced models.

\subsection{Characteristics of CuO NPs at obtained optimum conditions}

Formation of $\mathrm{CuO}$ NPs using walnut leaf extract could be evaluated based on their SPR, using UV-Vis spectral analysis. Figure 5 shows the broad emission peaks $\left(\lambda_{\max }\right)$ of the synthesized $\mathrm{CuO}$ NPs using optimum conditions, which the peak was achieved at $226 \mathrm{~nm}$. TEM analysis was done to monitor the morphology of the formed CuO NPs. TEM image of the synthesized NPs at obtained conditions indicated that the particles were monodispersed and spherical with mean particle size of $80 \mathrm{~nm}$ in diameter (Figure 6). XRD pattern of the formed CuO NPs using walnut leaf extract is shown in Figure 7. As clearly indicated in this figure, there were 11 peak positions with 




Wavelength (nm)

Figure 5: UV-Vis spectra of the mixture solution including synthesized CuO NPs at obtained optimum synthesis conditions.



Figure 6: SEM image of synthesized CUO NPs at obtained optimum conditions. 


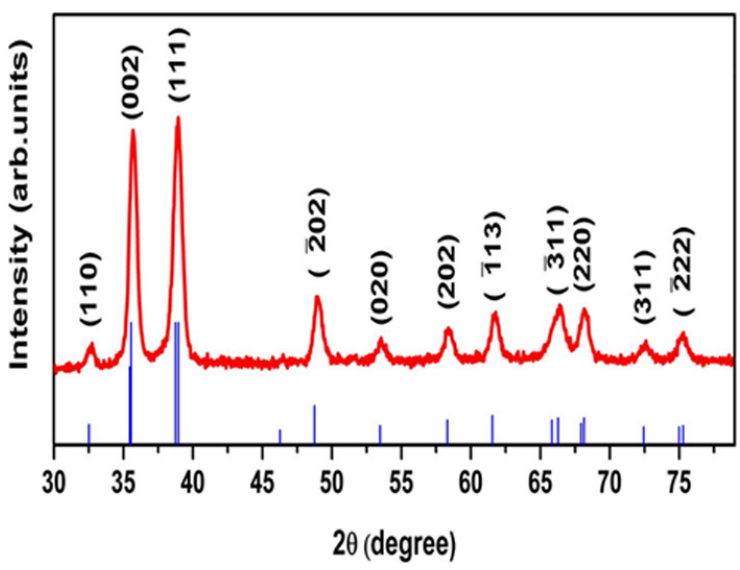

Figure 7: XRD pattern of synthesized CuO NPs at obtained optimum conditions.

different $2 \theta$ values which were in line with the finding of Ahmed et al. [24] and confirmed the fabrication of a crystalline structure for the CuO NPs.

\section{Conclusions}

As conclusion, present study indicated that walnut leaf extract due to several natural and bioactive compounds which those are capable to reduce copper ions and convert them into the NPs and stable the fabricated $\mathrm{CuO}$ NPs, could be effectively utilized in green synthesis of $\mathrm{CuO}$ NPs. Furthermore, RSM based on the Box behnken experimental design, could significantly evaluate the effects of synthesis parameters on the selected responses of the synthesized $\mathrm{CuO}$ NPs. Finally, the resulted green synthesized CuO NPs indicated high antioxidant (83.64\%) and antibacterial activities (MIC value of $1.78 \% \mathrm{w} / \mathrm{v}$ ), which these properties make the fabricated CuO NPs more applicable in various fields such as medicine and food packaging.

Acknowledgements: The authors appreciate the supports of Islamic Azad University-Tabriz branch to accomplish this paper.

\section{References}

[1] ljaz F., Shahid S., Khan S.A., Ahmad W., Zaman S., Green synthesis of copper oxide nanoparticles using Abutilon indicum leaf extract: antimicrobial, antioxidant and photocatalytic dye degradation activity. Trop. J. Pharm. Res., 2017, 16, 743-753.
[2] Din M.I., Rehan R., Synthesis, characterization, and applications of copper nanoparticles. Anal. Lett., 2017, 50, 50-62.

[3] Sravanthi M., Muni Kumar D., Usha B., Ravichandra M., Mahendra Rao M., Hemalatha K.P.J., Biological synthesis and characterization of copper oxide nanoparticles using Antigonon leptopus leaf extract and their antibacterial activity. Int. J. Adv. Res., 2016, 4, 589-602.

[4] Mohammadlou M., Maghsoudi H., Jafarizadeh-Malmiri H., A review on green silver nanoparticles based on plants: Synthesis, potential applications and eco-friendly approach. Int. Food Res. J., 2016, 23, 446-463.

[5] Mohammadlou M., Jafarizadeh-Malmiri H., Maghsoudi H., Hydrothermal green synthesis of silver nanoparticles using Pelargonium/Geranium leaf extract and evaluation of their antifungal activity. Green Process. Synth., 2017, 6, 31-42.

[6] Eskandari-Nojehdehi M., Jafarizadeh-Malmiri H., RahbarShahrouzi J., Optimization of processing parameters in green synthesis of gold nanoparticles using microwave and edible mushroom (Agaricus bisporus) extract and evaluation of their antibacterial activity. Nanotechnol. Rev., 2016, 5, 537-548.

[7] Fardsadegh B., Vaghari H., Mohammad-Jafari R., Najian Y., Jafarizadeh-Malmiri H., Biosynthesis, characterization and antimicrobial activities assessment of fabricated selenium nanoparticles using Pelargonium zonale leaf extract. Green Process. Synth., 2019, 8, 191-198.

[8] Sutradhar P., Saha M., Maiti D., Microwave synthesis of copper oxide nanoparticles using tea leaf and coffee powder extracts and its antibacterial activity. J. Nanostruct. Chem., 2014, 4, 86-91.

[9] Vanathi P., Rajiv P., Sivaraj R., Synthesis and characterization of Eichhornia-mediated copper oxide nanoparticles and assessing their antifungal activity against plant pathogens. Bull. Mater. Sci., 2016, 39, 1165-1170.

[10] Eshghi M., Vaghari H., Najian Y., Najian M.J., Jafarizadeh-Malmiri H., Berenjian A., Microwave-assisted green synthesis of silver nanoparticles using Juglans regia leaf extract and evaluation of their physico-chemical and antibacterial properties. Antibiotics, 2018, 7, 68-77.

[11] Vahidi A., Vaghari H., Najian Y., Najian M.J., Jafarizadeh-Malmiri H., Evaluation of three different green fabrication methods for the synthesis of crystalline $\mathrm{ZnO}$ nanoparticles using Pelargonium zonale leaf extract. Green Process. Synth., 2019, 8, 302-808.

[12] CLSI. Methods for dilution antimicrobial susceptibility tests for bacteria that grow aerobically; Approved Standard-Ninth Edition. CLSI document M07-A9. Wayne: Clinical and Laboratory Standards Institute, 2012.

[13] Amirkhani L., Moghaddas J., Jafarizadeh-Malmiri H., Candida rugosa lipase immobilization on magnetic silica aerogel nanodispersion. RSC Adv., 2016, 6, 12676-12687. 
[14] Ahmadi O., Jafarizadeh-Malmiri H., Jodeiri N., Eco-friendly microwave-enhanced green synthesis of silver nanoparticles using Aloe vera leaf extract and their physico-chemical and antibacterial studies. Green Process. Synth., 2018, 7, 231-140.

[15] Jafari N., Jafarizadeh-Malmiri H., Hamzeh-Mivehroud M., Adibpour M., Optimization of UV irradiation mutation conditions for cellulase production by mutant fungal strains of Aspergillus niger through solid state fermentation. Green Process. Synth., 2017, 6, 334-340.

[16] Eskandari-Nojehdehi M., Jafarizadeh-Malmiri H., Jafarizad A., Microwave accelerated green synthesis of gold nanoparticles using gum Arabic and their physico-chemical properties assessments. Z. Phys. Chem., 2018, 232, 325-343.

[17] Torabfam M., Jafarizadeh-Malmiri H., Microwave-enhanced silver nanoparticle synthesis using chitosan biopolymer: optimization of the process conditions and evaluation of their characteristics. Green Process. Synth., 2018, 7, 530-537.

[18] Eskandari-Nojedehi M., Jafarizadeh-Malmiri H., RahbarShahrouzi J., Hydrothermal green synthesis of gold nanoparticles using mushroom (Agaricus bisporus) extract: physico-chemical characteristics and antifungal activity studies. Green Process. Synth., 2018, 7, 38-47.

[19] Nottagh S., Hesari J., Peighambardoust S.H., Rezaei-Mokarram R., Jafarizadeh-Malmiri H., Development of a biodegradable coating formulation based on the biological characteristics of the Iranian Ultra-filtrated cheese. Biologia, 2018, 73, 403-413.

[20] Ghanbari S., Vaghari H., Sayyar Z., Adibpour M., JafarizadehMalmiri H., Autoclave-assisted green synthesis of silver nanoparticles using $A$. fumigatus mycelia extract and the evaluation of their physico-chemical properties and antibacterial activity. Green Process. Synth., 2018, 7, 217-224.

[21] Ahmadi O., Jafarizadeh-Malmiri H., Jodeiri N., Optimization of processing parameters for hydrothermal silver nanoparticles synthesis using Aloe vera leaf extract and estimation of their physico-chemical and antifungal properties. Z. Phys. Chem., 2018 (In press), DOI:10.1515/zpch-2017-1089.

[22] Anarjan N., Jaberi N., Yeganeh-Zare S., Banafshehchin E., Rahimirad A., Jafarizadeh-Malmiri H., Optimization of Mixing Parameters for $\alpha$-Tocopherol Nanodispersions Prepared Using Solvent Displacement Method. J. Am. Oil Chem. Soc., 2014, 91, 1397-1405.

[23] Anarjan N., Jafarizadeh-Malmiri H., Nehdi I.A., Sbihi H.M., Al-Resayes S.I., Tan C.P., Effects of homogenization process parameters on physicochemical properties of astaxanthin nanodispersions prepared using a solvent-diffusion technique. Int. J. Nanomed., 2015, 10, 1108-1119.

[24] Ahmed M., Alhadlaq H.A., Majeed Khan M.A., Karuppiah P., Al-Dhabi N.A., Synthesis, characterization, and antimicrobial activity of copper oxide nanoparticles. J. Nanomater., 2014, DOI:10.1155/2014/637858. 